\title{
Sarcoidosis aguda: a propósito de un caso
}

\author{
F. J. Zufía García, M. GarRido FernándeZ, \\ B. López Serrano, C. Refolio Abellá \\ Médicos de Familia. C. S. El Espinillo. Área 11. INSALUD. Madrid
}

\section{RESUMEN}

Las poliartralgias pueden deberse a diversas en fermedades de etiología y pronóstico muy variable. En ocasiones, se asocian lesiones cutáneas, que pueden orientar hacia determinadas patologías. El eritema nodoso puede ser la primera manifestación clínica de diversas enfermedades. Se caracteriza por la presencia de nódulos cutáneos eritematosos $y$ dolorosos, de 1 a 5 centímetros de diámetro, con marcado componente inflamatorio, localizados preferentemente en la superficie pretibial de las ex tremidades inferiores. Si se asocia con adenopatías hiliares bilaterales, constituye el síndrome de Löf gren, forma de sarcoidosis aguda de muy buen pro nóstico, con resolución espontánea en la mayoría de los casos.

Palabras clave: Sarcoidosis. Eritema nodoso. Sindrome de Löfgren.

Acute sarcoidosis: a case report

\section{ABSTRACT}

Poliarthralgias can be caused by different illnes ses with very variable etiology and prognosis. Sometimes these illnesses are associated with cuta neous lessions, that can help us in the diagnosis of the process. Erythema nodosum can be the initial clinical manifestation of diverse patologies. It is characterized by the presence of erythematous and painful subcutaneous nodules, from 1 to 5 centime ters in diameter, with important inflamation compo nent, preferably situated in the pretibial surface of the inferior extremities. The association with bila teral hiliar adenopathies is known as the Löfgren's syndrome, a kind of acute sarcoidosis with very good prognosis and spontaneous remissions in most cases.

Key words: Sarcoidosis. Erythema nodosum. Löfgren's syndrome.

\section{INTRODUCCIÓN}

En la práctica clínica diaria nos encontramos con frecuencia con pacientes que acuden por dolores articulares que afectan a varias articulaciones, sin signos inflamatorios. El diagnóstico diferencial debe basarse en el patrón temporal de la afectación, la presencia de fiebre, y la asociación con alteraciones sistémicas, así como la aparición de síntomas clave para el diagnóstico. La presencia de lesiones cutáneas puede ser determinante para el manejo de estos pacientes. El Eritema Nodoso (EN) puede ser la primera manifestación clínica de di- versas enfermedades. Se caracteriza por la presencia de nódulos cutáneos eritematosos y dolorosos, de 1 a 5 centímetros de diámetro, con marcado componente inflamatorio, localizados preferentemente en la superficie pretibial de las extremidades inferiores ${ }^{1}$. El color de las lesiones varía según el momento evolutivo: rojo vivo en su comienzo, cambia a rojo cianótico, y por último, tiene un componente purpúrico ${ }^{2}$. Más que como cuadro dermatológico, es importante como señal de toxicidad medicamentosa o de una enfermedad sistémica. Dentro de éstas, es de destacar por su elevada frecuencia, así como por su valor pronóstico, la 
sarcoidosis aguda, formando parte del síndrome de Löfgren. Presentamos un caso de poliartralgias y fiebre, asociado a eritema nodoso, y adenopatías hiliares.

\section{CASO CLÍNICO}

Mujer de 50 años, con antecedentes de neoplasia intracervical (C.I.N. grado III), tratada con conización, cistocele, mioma uterino y depresión reactiva a separación matrimonial. Consulta por dolores articulares generalizados de aparición reciente, coincidiendo con infección de vías respiratorias superiores, que no se acompaña de signos inflamatorios, por lo que se sospecha viriasis y se pauta tratamiento analgésico. A los siete días, acude a Urgencias del Hospital por aparición de lesiones maculopapulosas eritematosas, dolorosas, calientes, en ambas piernas, con edemas, acompañadas de fiebre de $38^{\circ} \mathrm{C}$. Se sospecha eritema nodoso, se realiza biopsia cutánea y se pauta ioduro potásico. Al día siguiente, vuelve a nuestra consulta, mostrándonos las lesiones descritas en miembros inferiores. El resto de la exploración física es normal. Puesto que el EN es con mucha frecuencia manifestación de enfermedades sistémicas, algunas de ellas graves, realizamos el estudio etiológico de éste: hemograma y bioquímica con perfil hepático normal, VSG 67 , proteína $\mathrm{C}$ reactiva 22 , factor reumatoide negativo, anticuerpos antinucleares negativos, aglutinaciones tíficas, paratíficas y bruce lla negativas, orina normal, radiografía de tórax: tórax óseo y campos pulmonares normales, ligero aumento de silueta cardiovascular con hilios grandes y densos, compatibles con adenopatías mediastínicas, ECG normal, mantoux $0 \mathrm{~mm}$, tres baciloscopias negativas. Ante la sospecha de sarcoidosis aguda y debido a la imposibilidad de solicitar TAC torácico desde nuestra consulta de AP, para confirmar la existencia de adenopatías hiliares, se derivó a un reumatólogo de referencia para su realización, con el siguiente resultado: adenopatías múltiples en mediastino y en ambos hilios pulmonares, el parénquima pulmonar no presenta alteraciones. Dos semanas después, presenta además tos seca y dolor esternal, manteniendo febrícula $\left(37,4{ }^{\circ} \mathrm{C}\right)$, con auscultación cardiopulmonar normal. La biopsia de lesiones cutáneas mostró: paniculitis septal (infiltrado perivascular con afectación de un septo). Se pautó tratamiento con indometacina $25 \mathrm{mg}$ cada 8 horas hasta la resolución de los síntomas. La tos desapareció en 2 meses, y las artralgias a los 7 meses del inicio del cuadro.

\section{JUICIO CLÍNICO}

Sarcoidosis aguda (S. de Löfgren).

\section{DISCUSIÓN}

Se trata de un cuadro de poliartralgia sin signos inflamatorios. El interrogatorio debe valorar la forma de comienzo, ritmo del dolor, incapacidad funcional, respuesta a tratamientos previos y presencia o no de síntomas acompañantes, como fiebre, pérdida de peso, síntomas cardiacos, respiratorios, digestivos o urinarios, manifestaciones dermatológicas u oculares ${ }^{3}$. Las quejas insidiosas y de larga evolución orientan a artrosis o fibromialgia. Las de comienzo reciente sugieren mayor probabilidad de enfermedad de mayor gravedad. El comienzo agudo obliga a descartar infección o empleo de fármacos (quinolonas, supresión de glucocorticoides). El dolor mecánico, que se alivia en reposo, y aumenta con los cambios de postura y a lo largo del día, orienta a fibromialgia o artrosis. El dolor inflamatorio, que empeora en reposo y mejora a lo largo del día, sugiere poliartritis, polimialgia reumática o colagenosis. Las alteraciones dermatológicas acompañantes pueden ser claves para el diagnóstico. En este caso se asocia a la aparición de lesiones compatibles con eritema nodoso.

Ante la sospecha clínica de EN, debemos confirmar el diagnóstico y tratar de localizar la etiología desencadenante del mismo. Entre las posibles etiologías del EN están los fármacos $(27,3 \%)$, sarcoidosis $(20,6 \%)$, infecciones $(16,3 \%$ postestreptocócicas y $6,3 \%$ tuberculosas del total), embarazo $(1,4 \%)$, enfermedades gastrointestinales y procesos malignos. Hay que tener en cuenta que en un $32,5 \%$ no se encuentra ninguna causa ${ }^{4}$. Para ello, se realizará el siguiente estudio: hemograma, VSG, bioquímica con perfil hepático, LDH, creatinina, ASLO, sistemático de orina, exudado faríngeo, baciloscopia y cultivo de esputo en medio de Lowenstein, radiografía de tórax, pruebas intradérmicas y en algunos casos biopsia de las lesiones ${ }^{2}$. Ésta sólo es necesaria en casos dudosos, ya que el diagnóstico es básicamente clínico ${ }^{1,4}$.

Nuestra paciente presenta fiebre, poliartralgias de comienzo reciente, y lesiones cutáneas compatibles con eritema nodoso. Realizamos el estudio etiológico de éste, recibiendo a los pocos días la radiografía de tórax con imágenes compatibles con adenopatías hiliares. Todo ello sugiere como primera probabilidad diagnóstica la sarcoidosis aguda. La confirmación de las adenopatías, así como la afectación o no del parénquima pulmonar es fundamental para el diagnóstico y el pronóstico de la misma, por lo que es precisa la realización de un TAC torácico, que en nuestro caso confirmó las adenopatías y la exclusión de afectación parenquimatosa, llegando al diagnóstico definitivo de sarcoidosis aguda. Löfgren, en 1946, describió por primera vez, un síndrome formado por la asociación de Eritema Nodoso (EN) y adenopatía hi- 
liar bilateral ${ }^{5}$. Constituye una forma de sarcoidosis aguda o subaguda, generalmente benigna, que con frecuencia se acompaña de poliartritis/poliartralgias y fiebre, más frecuente en mujeres adultas jóvenes (de 20-30 años de edad), y que se resuelve de

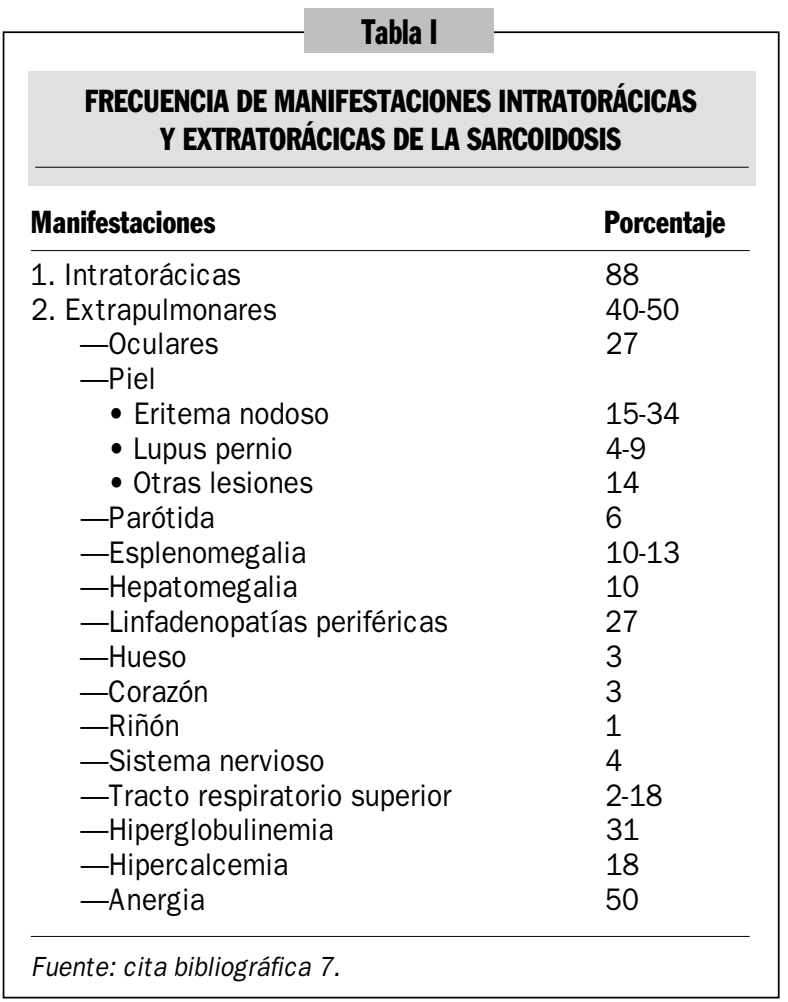

forma espontánea en pocos meses en la mayoría de los casos.

La sarcoidosis es una enfermedad granulomatosa multisistémica, de etiología desconocida, caracterizada por la acumulación en diferentes órganos, principalmente el pulmón, de granulomas formados por linfocitos $\mathrm{T}$ y macrófagos, que distorsionan la estructura normal del tejido en el que asientan ${ }^{6}$.

El diagnóstico se basa en la aparición de un cuadro clínico compatible, con afectación de al menos dos órganos, la evidencia histológica de granulomas epiteliodes no caseificantes, y la exclusión razonable de otras enfermedades granulomatosas ${ }^{8}$. Puede afectar a cualquier órgano, y los síntomas pueden deberse a enfermedad activa o residual. La frecuencia de afectación de cada órgano se describe en la tabla I. Hasta un $60 \%$ de pacientes pueden estar asintomáticos, descubriéndose de forma casual al realizar una radiografía de tórax, lo que suele predecir un curso benigno. Otros, como nuestro caso, tienen comienzo agudo, con eritema nodoso y adenopatías bilaterales. La fiebre, astenia, sudoración y pérdida de peso puede ocurrir en el $25 \%$ de casos. La afectación intratorácica, descrita en la tabla II, se da en el $88 \%$ de sujetos. El estudio inicial que debe realizar el médico de familia ante la sospecha de sarcoidosis será: hemograma y bioquímica hepática y renal, calcio, proteínas, orina con sedimento y calciuria, ECG, mantoux (comparar con previos), radiografía de tórax y pruebas funcionales respiratorias. Algunos autores aconsejan además el

Tabla II

\begin{tabular}{|c|c|c|c|c|c|}
\hline \multicolumn{6}{|c|}{ ESTADIOS DE LA SARCOIDOSIS } \\
\hline Estadio & Porcentaje & $\begin{array}{l}\text { Hallazgos radiológicos } \\
\text { de pacientes }\end{array}$ & $\begin{array}{l}\text { Test de función } \\
\text { pulmonar }\end{array}$ & Signos y síntomas & $\begin{array}{l}\text { Esperanza } \\
\text { de remisión (\%) }\end{array}$ \\
\hline 0 & $8-10$ & Normal & Normal & $\begin{array}{l}\text { Varía según sistemas } \\
\text { afectados }\end{array}$ & $\begin{array}{l}\text { La mayoría remi- } \\
\text { ten espontáneamente }\end{array}$ \\
\hline 1 & 51 & $\begin{array}{l}\text { Adenopatía hiliar } \\
\text { bilateral }\end{array}$ & $\begin{array}{l}\text { Normal, excepto } \\
\text { disminución de la } \\
\text { capacidad de difusión }\end{array}$ & $\begin{array}{l}\text { Mayoría asintomáticos } \\
\text { o sin síntomas } \\
\text { pulmonares }\end{array}$ & $\begin{array}{l}70-75 \% \text { remiten } \\
\text { en } 2 \text { años. } 10-15 \% \\
\text { progresan a estadio } 2\end{array}$ \\
\hline 2 & 29 & $\begin{array}{l}\text { Adenopatía hiliar } \\
\text { con infiltración } \\
\text { pulmonar difusa }\end{array}$ & $\begin{array}{l}\text { Patrón restrictivo, } \\
\text { a veces con altera- } \\
\text { ciones obstructivas }\end{array}$ & $\begin{array}{l}\text { Suaves en relación a } \\
\text { la severidad de los } \\
\text { hallazgos radiológicos }\end{array}$ & $\begin{array}{l}50 \% \text { remiten; } 25-30 \% \\
\text { persisten en estadio } \\
2 \text { o progresan al } 3\end{array}$ \\
\hline 3 & 12 & $\begin{array}{l}\text { Infiltración pulmonar } \\
\text { difusa sin adenopatías } \\
\text { hiliares. Generalmente } \\
\text { fibrosis. Puede haber } \\
\text { pérdidas de volumen, } \\
\text { quistes, bullas, } \\
\text { calcificaciones, } \\
\text { bronquiectasias }\end{array}$ & $\begin{array}{l}\text { Patrón restrictivo, } \\
\text { con alteraciones } \\
\text { obstructivas. Pueden } \\
\text { ser cambios muy } \\
\text { severos }\end{array}$ & $\begin{array}{l}\text { Variable. Pueden ser } \\
\text { mínimos (tos, disnea, } \\
\text { pérdida peso) o } \\
\text { severos (cor pulmonale, } \\
\text { hipertensión pulmonar, } \\
\text { fallo respiratorio) }\end{array}$ & $\begin{array}{l}30 \% \text { remiten } \\
\text { espontáneamente } \\
\text { en } 2 \text { años }\end{array}$ \\
\hline
\end{tabular}


test de difusión de CO. La biopsia es fundamental en el diagnóstico, debiéndose realizar siempre excepto en pacientes jóvenes con adenopatías hiliares bilaterales que están asintomáticos o presentan uveítis o EN (Löfgren) ${ }^{5,6,8}$. La enzima convertidora de la angiotensina sérica (ECA) se eleva en un 50$80 \%$ de pacientes, con valores más elevados si hay afectación intratorácica. Tiene un $40 \%$ de falsos negativos y un $10 \%$ de falsos positivos. Algunos autores completan el estudio con la prueba de Kvein, aunque es difícilmente obtenible y dudosamente inofensiva, por lo que no se hará de rutina. La prevalencia del EN en la sarcoidosis es muy variable, oscilando entre el 3 y el 53\% de los casos, debido a factores raciales, geográficos y genéticos. Es muy frecuente en Gran Bretaña, Escandinavia y en España. La aparición de EN en el curso de la sarcoidosis (ENS) hace que ésta se manifieste a edades más tempranas y con mayor predominio en mujeres. Se asocia con frecuencia con adenopatías hiliares bilaterales (estadio radiológico I), fiebre, artritis y/o artralgias, uveítis y prueba de mantoux negativa. Forma un complejo sintomático tan característico de sarcoidosis que para muchos autores no precisa la confirmación histológica ${ }^{5,6}$. La resolución espontánea del estadio I acontece entre el 60$80 \%$ de pacientes, cifra que se eleva al 95\% si consideramos sólo el síndrome de Löfgren. El ENS puede asociarse al estadio II, pero en este caso la resolución espontánea se reduce al $60 \%$ de casos. La evolución a la cronicidad es mucho mayor en los casos de sarcoidosis sin EN, lo que confirma el carácter benigno que el EN imprime a la sarcoidosis. Con frecuencia, el ENS se acompaña de síntomas generales, artralgias/artritis y uveítis. Salvo la uveítis, el resto acompañan al EN de otras etiologí- as. La sarcoidosis con EN se acompaña con menos frecuencia de síntomas respiratorios, lesiones cutáneas distintas del EN, afección ocular, adenopatías, hepatoesplenomegalia, afectación neurológica, alteraciones en el ECG y lesiones óseas, que la sarcoidosis sin EN.

Los corticoides han demostrado ser los fármacos más eficaces en el tratamiento de la sarcoidosis sintomática. En pacientes asintomáticos o con clínica respiratoria leve, con alta probabilidad de remisión espontánea (por ejemplo, EN), estaría indicada la observación durante un periodo de 2 a 6 meses sin tratamiento, ya que los efectos secundarios podrían ser más importantes que el posible beneficio de la terapia ${ }^{9}$. En los pacientes en los que se objetive deterioro clínico tras este intervalo, se debe comenzar con el tratamiento. Si no hubiera cambios, se debe individualizar la conducta. También estarían indicados de entrada los corticoides en caso de uveítis crónica, hipercalcemia, afectación cardiaca, afectación neurológica, afectación importante de cualquier órgano o fracaso de otros tratamientos. En nuestro caso, se pautó indometacina, como tratamiento sintomático de las artralgias, ya que no estaban indicados los corticoides. El ioduro potásico se empleó como tratamiento sintomático del eritema nodoso.

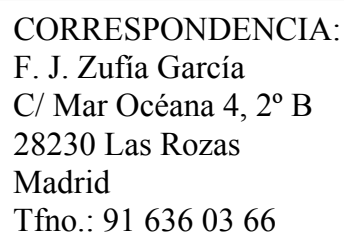

\section{Bibliografía}

1. Esquerrá MT, Molina S, Deniel J. Eritema nudoso. JANO 1999; 57: 39-42.

2. Lado F, Ferreiro MJ, Matilla L, Sánchez J, Martínez J, Barrio E. Un eritema nudoso. Med Integral 1998; 31: 185-9.

3. Naranjo A. Un paciente con dolor poliarticular. Med Integral 1998; 32: 81-5.

4. Atanes A, Sánchez-Bursón JM. EN. Rev Esp Reumatol 1989; 16: 166-71.

5. Atanes A, Toro J, Gómez N, Aspe B, Graña J, Burson J, et al. Estudio de 94 casos de sarcoidosis con especial referencia al eritema nudoso. Rev Clin Esp 1992; 191: 65-70.
6. Muñoz RM, Yebra M, López E, Vargas JA. Sarcoidosis. Medicine 1997; 54: 2446-52.

7. Belfer M, Wayne R. Sarcoidosis: a Primary Care Review. Am Fam Physician 1998; 58: 2041-50.

8. Johns C, Michele TM. The clinical management of sarcoidosis. A 50-year experience at the Johns Hopkins Hospital. Medicine Baltimore 1999; 78: 65-111.

9. Judson M. An approach to the treatment of Pulmonary Sarcoidosis with corticosteroids. The six phases of treatment. Chest 1999; 115: 1158-65. 